\title{
EFFECTS OF POLYPHENOL SUPPLEMENTATION ON THE IN VITRO ANTIOXIDANT POTENTIAL OF CITRUS JUICES
}

\author{
Agnieszka Synowiec-Wojtarowicz ${ }^{1}$, Yoana Kiselova ${ }^{2}$, Dobri Ivanov ${ }^{3}$, Diana Ivanova ${ }^{2}$, \\ Włodzimierz Bialik ${ }^{4}$, Magdalena Kimsa-Dudek ${ }^{1}$, Katarzyna Pawłowska-Góral ${ }^{1}$ \\ ${ }^{1}$ Department of Molecular Biology, Faculty of Pharmacy, SUM Katowice, Poland \\ ${ }^{2}$ Department of Biochemistry, Molecular Medicine and Nutrigenomics, \\ Faculty of Pharmacy, Medical University of Varna, Bulgaria \\ ${ }^{3}$ Department of Biology, Faculty of Pharmacy, Medical University of Varna, Bulgaria \\ ${ }^{4}$ Department of Pharmacoeconomics and Social Pharmacy, School of Pharmacy, \\ SUM Katowice, Poland
}

\begin{abstract}
Food preparations containing substances with antioxidant activity have considerably aroused the interest of consumers, which has led to the marketing of a number of dietary supplements such as resveratrol, hesperidin or chlorogenic acid. On the other hand, natural antioxidants present in citrus fruit juices, together with dietary supplements, could contribute to an enhancement of their antioxidant properties and, in turn, alter their physiological impacts. The aim of the study was to evaluate the effect of supplementation of freshly squeezed citrus juices with resveratrol, hesperidin and chlorogenic acid on the in vitro antioxidant potential of the juices. The solutions of juices, selected polyphenols and the mixtures of solutions of juices and solution of polyphenols were analyzed to determine total polyphenol concentration using the Fast Blue method and total antioxidant potential by the ABTS method. As a result three different types of interactions were established: synergistic of chlorogenic acid which additionally increased the antioxidant potential by 35 - 38\% over theoretical predictions, additive - after addition of resveratrol, and antagonistic - in the case of hesperidin. While the antioxidant potential was substantially higher in the mixture of mandarin juice with chlorogenic acid, the measured total polyphenol concentration decreased. Also a considerable decrease in the concentration of polyphenols was measured in the mixtures of mandarin and orange juices with resveratrol, all these possibly due to the sensitivity to other substances present in juices of the analytical method applied. A correlation between antioxidant potential of all examined solutions and total polyphenol concentration was found $\left(r^{2}=0.8879\right)$.
\end{abstract}

Keywords: antioxidants, antioxidant supplementation, polyphenols, citrus juices

Address for correspondence:

Diana Ivanova

Faculty of Pharmacy

Medical University of Varna

84 Tzar Osvoboditel Blvd

e-mail:divanova@mu-varna.bg

Received: May 21, 2018

Accepted: May 31, 2018

\section{INTRODUCTION}

Over the last years, there has been a very dynamic increase in the consumption of dietary supplements. However, advertising campaigns often create a false image of these products, assigning them curative properties. Considerable interest among consumers is aroused by preparations containing substances possessing antioxidant activity which has 
Agnieszka Synowiec-Wojtarowicz, Yoana Kiselova, Dobri Ivanov et al.

led to the marketing of a number of dietary supplements containing polyphenols such as resveratrol, hesperidin or chlorogenic acid. According to the latest review (1) resveratrol has therapeutic potential for cardiovascular protection and prevention similar to other known natural antioxidants such as co-enzyme Q10 and to omega-3 fatty acid. Nevertheless, it remains a pharmaceutical challenge to effectively deliver resveratrol systemically to increase its oral bioavailability (2). Hesperidin is also an interesting compound according to a recent review on preclinical studies and clinical trials demonstrating therapeutical effects in various diseases (3). The wide range of potential health benefits of chlorogenic acid, including its anti-diabetic, anti-carcinogenic, anti-inflammatory and anti-obesity impacts have recently been reviewed as well (4).

On the other hand, antioxidants are present in citrus fruit juices (5), which taken together with dietary supplements could contribute to an enhancement of their antioxidant properties. When foods are consumed together, the total antioxidant capacity of food mixtures may be modified via synergistic, additive, or antagonistic interactions among these components, which may in turn alter their physiological impacts (6).

\section{AIM}

The aim of the study was to evaluate the effect of supplementation of freshly squeezed citrus juices with selected polyphenols, resveratrol, hesperidin and chlorogenic acid, on the in vitro antioxidant potential of the juices.

\section{MATERIALS AND METHODS}

The juices from peeled off fruits - limes (L), mandarins $(\mathrm{M})$ or oranges $(\mathrm{O})$ were obtained using a mechanical squeezer (Angel, Angel Co., South Korea), then centrifuged (Centrifuge MPW 365, Precision Mechanics, Warsaw, Poland) and diluted (1:20, $\mathrm{v} / \mathrm{v}$ ) in distilled water. Even though methanol is toxic to humans, for the needs of the current study polyphenols were disolved in methanol, which is the best solubilizer for the tested substances, to ensure the presence of a maximum amount of the substance. Twenty milligrams of appropriate polyphenol: chlorogenic acid $(\mathrm{Ch})$, resveratrol $(\mathrm{R})$, or hesperidin $(\mathrm{H})$ ( Sigma - Aldrich, Germany) were dissolved in $10 \mathrm{ml}$ of methanol (Chempur, Piekary Śląskie, Poland), and diluted $(1: 20, \mathrm{v} / \mathrm{v})$ in methanol. Then the diluted juices were mixed with diluted polyphenols in a ratio 1 to 1. Finally, all the obtained solutions of juices, selected polyphenols and the mixtures of solutions of juices and solution of selected polyphenols were analyzed in order to determine total polyphenol concentration using the Fast Blue method as described by Medina (7). The method is based on interactions of phenolics with Fast Blue BB diazonium salt in alkali $\mathrm{pH}$, forming azo complexes, with the absorbance measured at $420 \mathrm{~nm}$ after $60 \mathrm{~min}$. The total polyphenol concentration was expressed as a mean in mg of gallic acid equivalents ( $\mathrm{g} / \mathrm{l}$ of sample).

Total antioxidant potential was analyzed using the method of Re et al. (1999) (8), as described earlier (9). In brief, the method is based on the consumption by available antioxidants of the preformed in the presence of potassium persulphate $\left[2,2^{\prime}\right.$-azinobis (3-ethylbenzothiazoline-6-sulfonic acid)] cation radical (ABTS+), the absorption maximum of which is at $734 \mathrm{~nm}$. The extent of discoloration of the bluegreen color as percentage inhibition of the ABTS+ radical correlates to the concentration of antioxidants and is calculated relative to the reactivity of Trolox, a water-soluble analog of vitamin E sold by Hoffman-LaRoche, (6-hydroxy-2,5,7,8-tetramethylchroman-2-carboxylic acid) by standard. The total antioxidant potential was expressed as Trolox equivalent antioxidant capacity (mmol/l of sample).

SP-830 Plus (Metertech, Taiwan) spectrophotometer was used to perform the measurements. All determinations were made in four replications. Results are present as mean \pm SD.

The hypothesis concerning the compatibility of the distribution of a given parameter with the normal distribution was verified by the Shapiro-Wilk test using the STATISTICA v. 10.0 computer program (StatSoft, Tulsa, Oklahoma). The comparisons between the two groups were made using Student's $t$ test for independent trials. The relationship between the two variables was determined using the Pearson's linear correlation coefficient. P value $<0.05$ was considered as statistically significant.

\section{RESULTS AND DISCUSSION}

The values of the antioxidant potential of the solutions of juices and selected polyphenols as well as 
experimentally determined total polyphenol concentrations are listed in Table 1.

The data in the Table 1 shows that the best antioxidant potential and the highest polyphenol concentration was measured for the solution of resvera-

Table 1. Antioxidant potential (ABTS) and polyphenol concentrations of citrus juices and selected polyphenol solutions

\begin{tabular}{lcc} 
& $\begin{array}{c}\text { Antioxi- } \\
\text { dant poten- } \\
\text { tial ABTS } \\
{[\mathrm{mmol} / \mathrm{l}] \pm \text { SD }}\end{array}$ & $\begin{array}{c}\text { Total } \\
\text { Polyphenols } \\
{[\mathrm{g} / \mathrm{l}] \pm \mathrm{SD}}\end{array}$ \\
Lime & $3.02 \pm 0.23$ & $3.59 \pm 0.23$ \\
Mandarin & $4.02 \pm 0.32$ & $1.87 \pm 0.21$ \\
Orange & $6.14 \pm 0.54$ & $2.86 \pm 0.22$ \\
Chlorogenic acid & $3.77 \pm 0.36$ & $3.91 \pm 0.25$ \\
Resveratrol & $23.93 \pm 0.78$ & $11.9 \pm 0.29$ \\
Hesperidin & $2.73 \pm 0.36$ & $3.09 \pm 0.31$ \\
\hline
\end{tabular}

trol. The solutions of orange juices exhibited the best antioxidant potential, however, the highest polyphenol concentration was measured for the solution of lime juice. Nevertheless, correlation between the antioxidant potentials and the total polyphenol concentrations seemed to be rather good $\left(\mathrm{r}^{2}=0.8349\right)$.

Table 2 summarizes the data obtained for mixtures of juices with polyphenols solutions. The columns labelled "calculated" show the numbers calculated from the data in the Table 1, taking into account that the juice solutions and polyphenol solutions were mixed at the ratio 1 to 1 . The columns labelled "measured" present the values obtained in real experiments when the solutions of juices were mixed with the solutions of polyphenols and then analyzed.

The data in Table 2 shows considerable discrepancies between the calculated and experimental results, which indicates that in the majority of cases interactions between the added polyphenols and the constituents of the juices do exist. In order to ex-

Table 2. Summary of the calculated and experimentally obtained results of antioxidant potential (ABTS) and polyphenol concentration for mixtures of juices and polyphenol solutions;

* - difference is statistically significant, $n s$ - lack of statistical significance

\begin{tabular}{|c|c|c|c|c|c|c|}
\hline \multirow[b]{2}{*}{ Juice+polyphenol } & Calculated & Measured & \multirow[b]{2}{*}{$\begin{array}{l}\text { Statictical } \\
\text { signifi- } \\
\text { cance }\end{array}$} & \multirow{2}{*}{$\begin{array}{l}\text { Calculated } \\
\text { Total Poly- } \\
\text { phenols } \\
\text { [g/l] }\end{array}$} & \multirow{2}{*}{$\begin{array}{c}\text { Measured } \\
\text { Total Poly- } \\
\text { phenols } \\
\text { [g/l] }\end{array}$} & \multirow[b]{2}{*}{$\begin{array}{l}\text { Statictical } \\
\text { significance }\end{array}$} \\
\hline & $\begin{array}{c}\text { Antioxidant } \\
\text { potential } \\
\text { ABTS } \\
{[\mathrm{mmol} / \mathrm{l}]}\end{array}$ & $\begin{array}{c}\text { Antioxidant } \\
\text { potential } \\
\text { ABTS } \\
{[\mathrm{mmol} / \mathrm{l}]}\end{array}$ & & & & \\
\hline $\begin{array}{l}\text { Lime }+ \text { chloroge- } \\
\text { nic acid }\end{array}$ & $3.40 \pm 0.21$ & $\uparrow 4.70 \pm 0.36$ & * & $3.75 \pm 0.14$ & $\uparrow 4.11 \pm 0.35$ & * \\
\hline Lime + resveratrol & $13.48 \pm 0.58$ & $13.74 \pm 0.62$ & ns & $7.75 \pm 0.41$ & $8.19 \pm 0.37$ & ns \\
\hline Lime + hesperidin & $2.88 \pm 0.11$ & $\downarrow 2.49 \pm 0.24$ & * & $3.35 \pm 0.12$ & $\downarrow 2.67 \pm 0.31$ & * \\
\hline $\begin{array}{l}\text { Mandarin }+ \\
\text { chlorogenic acid }\end{array}$ & $3.90 \pm 0.23$ & $\downarrow 5.27 \pm 0.28$ & * & $2.89 \pm 0.14$ & $\downarrow 2.54 \pm 0.13$ & * \\
\hline $\begin{array}{l}\text { Mandarin + } \\
\text { resveratrol }\end{array}$ & $13.98 \pm 0.53$ & $13.52 \pm 0.65$ & ns & $6.89 \pm 0.31$ & $\downarrow 5.87 \pm 0.28$ & * \\
\hline $\begin{array}{l}\text { Mandarin + } \\
\text { hesperidin }\end{array}$ & $3.38 \pm 0.22$ & $\downarrow 2.61 \pm 0.23$ & * & $2.48 \pm 0.18$ & $\downarrow 1.88 \pm 0.26$ & * \\
\hline $\begin{array}{l}\text { Orange }+ \\
\text { chlorogenic acid }\end{array}$ & $4.96 \pm 0.51$ & $\uparrow 6.85 \pm 0.64$ & * & $3.39 \pm 0.17$ & $\uparrow 3.96 \pm 0.24$ & * \\
\hline $\begin{array}{l}\text { Orange }+ \\
\text { resveratrol }\end{array}$ & $15.04 \pm 0.39$ & $15.01 \pm 0.59$ & ns & $7.38 \pm 0.36$ & $\downarrow 6.21 \pm 0.29$ & * \\
\hline $\begin{array}{l}\text { Orange + } \\
\text { hesperidin }\end{array}$ & $4.44 \pm 0.1$ & $\downarrow 4.16 \pm 0.13$ & * & $2.98 \pm 0.19$ & $\downarrow 2.63 \pm 0.17$ & * \\
\hline
\end{tabular}


Agnieszka Synowiec-Wojtarowicz, Yoana Kiselova, Dobri Ivanov et al.

amine them more thoroughly, the next two figures (Fig. 1 and Fig. 2) present data in graphs in which the baseline stands for calculated values and the differences in plus and in minus can be clearly seen.

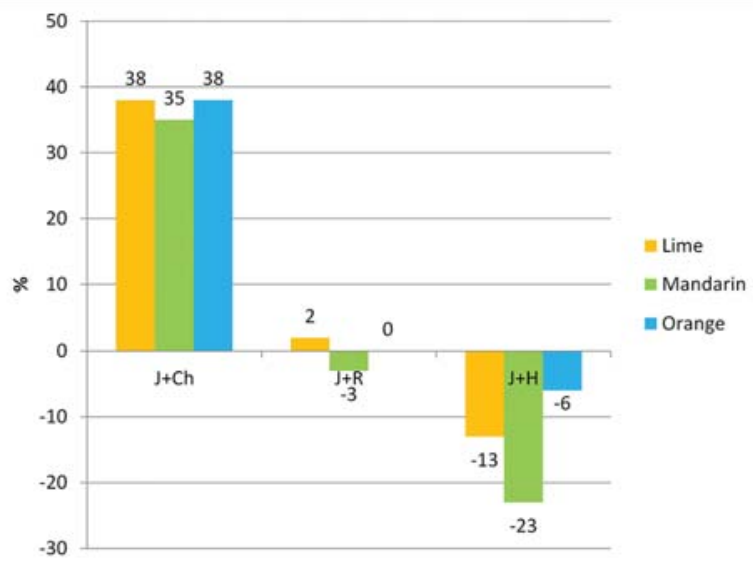

Fig. 1. Difference [\%] (measured vs. calculated) in the antioxidant potential of solutions of citrus juices $(J)$ mixed with chlorogenic acid $(J+C h)$, resveratrol $(J+R)$ and hesperidin $(J+H)$, respectively

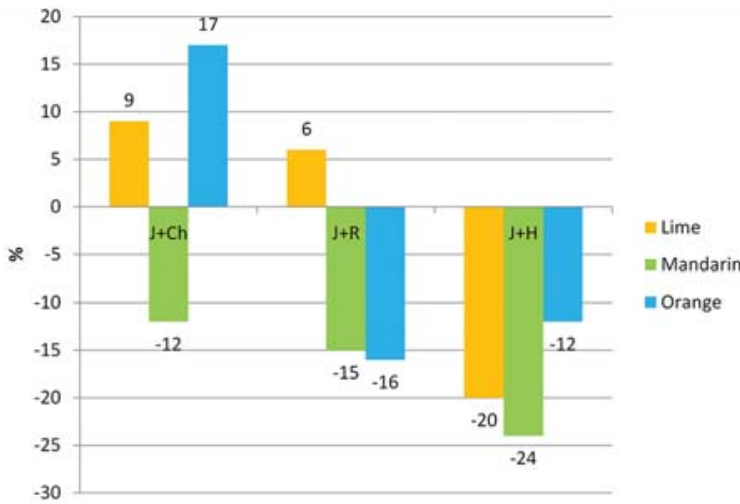

Fig. 2. Differences [\%] (measured vs. calculated) in the total polyphenol concentration of solutions of citrus juices (J) mixed with chlorogenic acid $(J+C h)$, resveratrol $(J+R)$ and hesperidin $(\mathrm{J}+\mathrm{H})$

Three different types of interactions are visualized on Fig. 1 and are as follows: synergistic - when addition of chlorogenic acid to the juices solutions increases the antioxidant potential by $35-38 \%$ over theoretical predictions, additive - after addition of resveratrol, and antagonist - in the case of hesperidin. These results confirm the previously established finding of Wang et al. (2011) (6). The results presented on Fig. 2 were in this line only in case of hesperidin. The findings about the mixture of mandarin juice with chlorogenic acid are especially surprising because the measured total polyphenol concentration decreased while the antioxidant potential was substantially higher. Also, the considerable decrease in the measured polyphenol concentrations in the mixtures of mandarin and orange juices with resveratrol is difficult to explain. It is therefore possible that the analytical method applied is sensitive to other substances present in juices. Nevertheless, correlation between the antioxidant potential of all examined solutions and the total polyphenol concentration was rather good $\left(\mathrm{r}^{2}=0.8879\right)$, as presented on the Fig. 3.

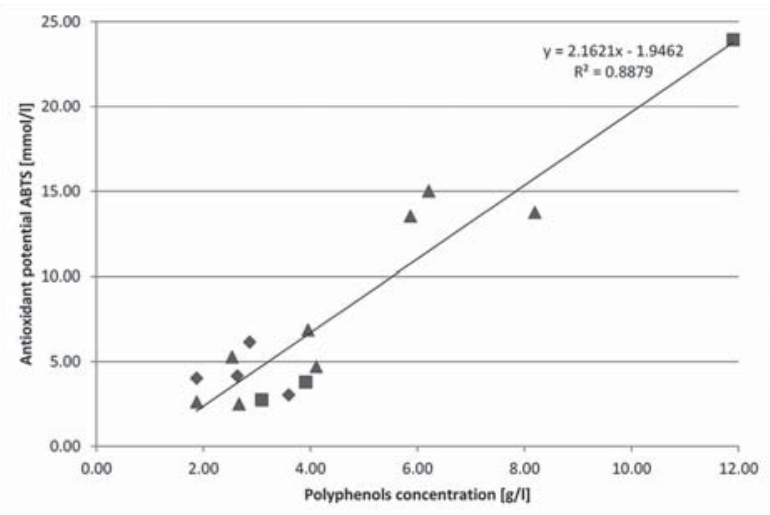

Fig. 3. Correlation between the antioxidant potential and total polyphenol concentration of solutions of selected polyphenols (squares), juices (diamonds) and mixtures of juices and solutions of seleceted polyphenols (triangles)

\section{CONCLUSION}

1. The antioxidant potential of the juices was modified via synergistic interactions after addition of chlorogenic acid, additive interactions with resveratrol and antagonistic ones with hesperidin.

2. Antioxidant potential generally correlates well with measured total polyphenol concentration; however, some derogations, which could be related to the applied analytical methods, were observed.

Perhaps optimized mixtures of fresh juices and natural extracts containing a variety of antioxidants can be effectively used in different combina- 
tions as substituents of synthetic antioxidants and this should be explored in future studies.

Acknowledgements:

The authors thank Ewa Kurzeja PhD for her assistance with the experimental section.

\section{REFERENCES}

1. Yeung PK. Therapeutic potential of resveratrol for cardiovascular protection. Cardiovasc Pharm. 2017 Open Access 6:e134. doi: 10.4172/2329-6607.1000e134.

2. Francioso A, Mastromarino P, Masci A, D'Erme M, Mosca L. Chemistry, stability and bioavailability of resveratrol. Med Chem. 2014; 10(3): 237-45.

3. Li C, Schluesener $\mathrm{H}$. Health-promoting effects of the citrus flavanone hesperidin. Crit Rev Food Sci Nutr. 2017; 57(3):613-31. doi: 10.1080/10408398.2014.906382.

4. Tajik N, Tajik M, Mack I, Enck P. The potential effects of chlorogenic acid, the main phenolic components in coffee, on health: a comprehensive review of the literature. Eur J Nutr. 2017; 56(7):2215-2244. doi: 10.1007/s00394-017-1379-1.

5. Okwu DE. Citrus fruits: a rich source of phytochemicals and their roles in human health. Int $J$ Chem Sci. 2008; 6(2): 451-71.

6. Wang S, Meckling KA, Marcone MF, Kakuda Y, Tsao R. Synergistic, additive, and antagonistic effects of food mixtures on total antioxidant capacities. J Agric Food Chem. 2011; 59(3):960-8. doi: 10.1021/jf1040977.

7. Medina MB. Simple and rapid method for the analysis of phenolic compounds in beverages and grains. J Agric Food Chem. 2011; 59(5): 1565-71. doi: 10.1021/ff103711c.

8. Re R, Pellegrini N, Proteggente A, Pannala A, Yang $\mathrm{M}$, Rice-Evans C. Antioxidant activity applying an improved ABTS radical cation decolorization assay. Free Rad Biol Med. 1999; 26(9-10):1231-7. doi: 10.1016/S0891-5849(98)00315-3.

9. Kiselova $Y$, Ivanova $D$, Chervenkov $T$, Gerova $D$, Galunska B, Yankova T. Correlation between the in vitro antioxidant activity and polyphenol content of aqueous extracts from Bulgarian herbs. Phytother Res. 2006; 20(11):961-5. doi: 10.1002/ptr.1985. 